\title{
Factores que Afectan la Precisión de la Estimación del Esfuerzo en Proyectos de Software Usando Puntos de Caso de Uso
}

\author{
Luis Morales Huanca ${ }^{1}$, Sussy Bayona Oré \\ luis.morales2@unmsm.edu.pe,sbayonao@hotmail.com \\ ${ }^{1}$ Unidad de Posgrado de la Facultad de Sistemas e Informática, Universidad Nacional Mayor de San Marcos \\ (UNMSM), Av. Germán Amézaga s/n, Lima, Perú.
}

DOI: 10.17013/risti.21.18-32

\begin{abstract}
Resumen: El éxito de un proyecto de desarrollo de software depende de que el producto obtenido cumpla con las especificaciones del usuario y se termine dentro del plazo y con el presupuesto establecido. Muchos proyectos de software presentan el problema de no estar siendo desarrollados dentro del plazo planificado debido a una mala valoración del esfuerzo o duración del proyecto de software. La presente investigación tiene como objetivo determinar los factores que afectan la precisión de la estimación del esfuerzo en proyectos de software, usando Puntos de Caso de Uso como método de estimación. Un total de 37 estudios primarios fueron seleccionados. Los resultados muestran que los factores ambientales, la complejidad de los casos de uso, la falta de estandarización de los casos de uso, factores técnicos y contabilización de las transacciones son algunos de los factores que afectan la precisión del método de Puntos de Caso de Uso.
\end{abstract}

Palabras-clave: Proyecto de Desarrollo de Software; Puntos de Caso de Uso; Estimación de esfuerzo.

\section{Factors Affecting the Accuracy of Effort Estimation in Software Projects Using Use Case Points}

\begin{abstract}
The success of a software development project depends on that the retrieved product complies with the user's specifications and to be completed within the time and within the budget established. Many software projects show the problem about that they are not being developed within the time set due to a bad assessment of the effort or duration of the software project. This research has the objective of determining the factors that affect the accuracy of the effort estimation in software projects using the Use Case Points as estimation method. A total of 37 primarily studies were selected. The results show that the environmental factors, the use cases complexity, the lack of use case standardization, technical factors and counting transactions are some of the factors that affect the accuracy of the Use Case Points method.
\end{abstract}

Keywords: Software development projects; Use Case Points; Effort estimation. 


\section{Introducción}

Para la industria del software predecir cuál será el esfuerzo requerido para un proyecto de software tan tempranamente como sea posible es de suma importancia (Ayyldız \& Koçyiğit, 2014). La estimación del esfuerzo es una métrica fundamental en proyectos de software (Chavarría, Oré \& Pastor, 2016), pudiendo ser formalizado mediante la creación de un método (Flores-Rios, Pino, Ibarra-Esquer, González-Navarro \& Rodríguez-Elías, 2014). Existen métodos de estimación de esfuerzo que son adecuados para diferentes ambientes, software, etc., pero todavía no están dando mejores resultados siempre debido a algunos problemas o factores (Saroha \& Sahu, 2015). Muchos investigadores han utilizado el método basado en tecnología orientado a objetos conocido como Puntos de Caso de Uso para la estimación de esfuerzo en el desarrollo de un proyecto de software. Los casos de uso son la base del método de Puntos de Caso de Uso, mediante el cual se puede documentar los requerimientos de un sistema en términos de actores y casos de uso, proporcionando uno o más escenarios que indican cómo debería interactuar el sistema con el usuario o con otro sistema para conseguir un objetivo específico. La estimación del esfuerzo en proyectos de software mediante el uso del método de Puntos de Caso de Uso, consiste en un método de estimación del tiempo de desarrollo de un proyecto de software mediante la asignación de "pesos" a un cierto número de factores que lo afectan, para que luego a partir de esos factores se pueda contabilizar el tiempo total estimado para el proyecto de software en cuestión (Recalde \& Lopez, 2010; Vasconcelos et al., 2017).

En el presente artículo se realiza una revisión de literatura exhaustiva orientada a determinar los factores que afectan la precisión en la estimación del método de Puntos de Caso de Uso, para lo cual se tomará como referencia estudios realizados entre los años 2010 al 2016. En la sección 2 se presenta el marco teórico. En la sección 3 se presenta la revisión de la literatura. En la sección 4 se presenta el informe de la revisión. En la sección 5 se presenta el anexo del listado de artículos primarios y finalmente en la sección 6 se presenta las conclusiones.

\section{Marco Teórico}

\subsection{Estimación de Esfuerzo}

Trendowicz y Jeffery definen la estimación de esfuerzo como una combinación de persona y tiempo, refiriéndose al tiempo de trabajo enteramente productivo que una persona debería necesitar para completar un cierto trabajo (Trendowicz \& Jeffery, 2014). La estimación del esfuerzo a invertir en proyectos de desarrollo de software es vital para la planificación de proyectos ya que nos da una medida del tiempo y costo estimado durante el ciclo de vida del proyecto de software. Sin embargo, la estimación de costos y esfuerzos sigue siendo una de las tareas más difíciles en la gestión de un proyecto de software, a pesar de que existen técnicas que permiten realizar esta tarea (Pow-Sang, 2004). La duración de las tareas y estimaciones de esfuerzo se determinan durante la etapa de planificación del proyecto y proporcionan la base para la posterior planificación, control y toma de decisiones. Las estimaciones son, en efecto, conjeturas respecto al rendimiento futuro basado en el conocimiento disponible. Como tal, su exactitud se ve afectada por el grado de incertidumbre con respecto a la tarea que se estima. La incertidumbre está asociada, entre otras cosas, con las definiciones de los requisitos, elección de la solución 
tecnológica, innovación de necesidad, y las características del cliente (Morgenshtern, Raz \& Dvir, 2007). Luego, la parte más crítica y crucial de estimación de software es cuando se requiere la estimación en las primeras etapas del ciclo de vida del software en el que el problema a resolver no ha sido aun completamente revelado. La realización de una estimación temprana del esfuerzo en proyecto de software ayuda a que los proyectos sean administrados de manera eficiente y ayuda a los gerentes a estimar el esfuerzo, planificar y costear el proyecto. Esto a su vez, permite a los gerentes realizar una oferta efectiva en proyectos de software (Nassif, Capretz \& Ho, 2010).

\subsection{Puntos de Caso de Uso}

En la actualidad la mayoría de proyectos de software desarrollados están utilizando la tecnología orientada a objetos para el desarrollo de proyectos de software y el método de puntos de caso de uso para la estimación de esfuerzo (Kirmani \& Wahid, 2015). Puntos de Caso de Uso es utilizado para predecir el esfuerzo en horas hombre que toma desarrollar un proyecto de software, propuesto originalmente por Karner, quien lo define como un modelo que da una estimación del tamaño del esfuerzo para desarrollar un sistema que puede ser mapeado a horas-hombre para completar diversas fases del objetivo o completar todo el proyecto (Karner, 1993).

El método de Puntos de Caso de Uso presenta fortalezas y debilidades. Algunas de las fortalezas de este método son: (1) que puede ser utilizado en una fase temprana para estimar el esfuerzo, y es medido en base a un modelo de casos de uso que define el alcance funcional del sistema de software a ser desarrollado (Kusumoto, Matukawa, Inoue, Hanabusa \& Maegawa, 2005), (2) es versátil y extensible a una variedad de proyectos de desarrollo y de pruebas, fácil de aprender y rápido para aplicar (Clemmons, 2006), (3) es conocido y ampliamente aceptado ya que utiliza dos prácticas comunes en la industria: el paradigma orientado a objetos y casos de uso para describir los requisitos de funcionalidad (Wang, Yang, Zhu \& Chen, 2009). Sin embargo, también presenta algunas debilidades que están asociadas a los factores que afectan la precisión de la estimación del método de Puntos de Caso de Uso las cuales se muestran a continuación: (1) la primera versión del método de Puntos de Caso de Uso carece de validación y examinación sobre su fiabilidad para las organizaciones de software (Azzeh \& Nassif, 2016), (2) incertidumbre asociada a los factores de costo y clasificación abrupta de los casos de uso (Wang, Yang, Zhu \& Chen, 2009), (3) necesidad de calibración respecto a los niveles de complejidad de casos de usos y clasificación de actores (Nassif, Capretz \& Ho, 2010), (4) carencia de información acerca del conteo del número de actores y de casos de uso y desfase de los factores técnicos y ambientales (Singh \& Sahoo, 2012) y (5) discusión al asumir que la relación entre el esfuerzo del software y el tamaño sea lineal (Satapathy \& Rath, 2014). Como resultado de las debilidades del método de Puntos de Caso de Uso existen varios factores que afectan la precisión en la estimación de este método. El estudio de estos factores se analiza a más detalle en la sección 4 (Informe de la Revisión).

\section{Revisión de la Literatura}

La revisión de la literatura se refiere a la estructura y reglas que componen la presente investigación. Esta revisión busca identificar los factores que afectan la estimación de la 
técnica de Puntos de Caso de Uso en cuanto a su precisión. La revisión de la literatura realizada consta de los siguientes pasos: identificación de las preguntas de investigación, diseño de la estrategia de búsqueda, conducción de la revisión, síntesis de datos, resultados, y discusiones (ver Figura 1).

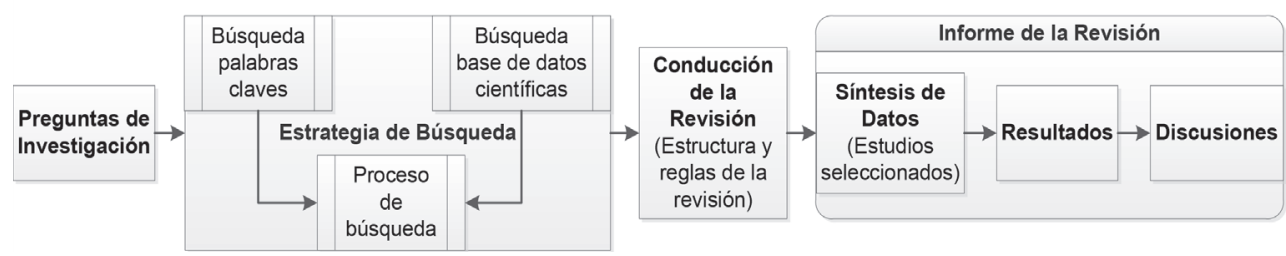

Figura 1 - Pasos realizados en Revisión de Literatura.

\subsection{Preguntas de Investigación}

Las preguntas de investigación que se plantean son: (1) ¿Qué factores o limitaciones afectan la precisión de la estimación del método de Puntos de Caso de Uso?, (2) ¿Cuáles son los futuros trabajos a realizar respecto al método de Puntos de Caso de Uso?

\subsection{Estrategia de Búsqueda}

Las siguientes fuentes fueron seleccionadas para llevar a cabo el proceso de búsqueda: ACM Digital Library, IEEE Xplore, Science Direct - Elsevier, Springer Link, Taylor and Francis, y Google Scholar. Se definieron las palabras claves para encontrar estudios relevantes en base a los títulos, resumen y contenido de artículos dentro del periodo 2010-2016. Las palabras claves utilizadas son: "Use Case Point" OR "Use Case Points" OR "Puntos de casos de usos" OR "Puntos de casos de uso" OR "Puntos de caso de uso" OR "Puntos de caso de usos" OR "Punto de casos de usos" OR "Punto de casos de uso".

\section{3·3.Conducción de la Revisión}

La conducción de la revisión hace referencia a la estructura y reglas para llevar a cabo la revisión. A continuación se mencionan los pasos utilizados para conducir la presente revisión (ver Figura 2): (1) Se identificó estudios relevantes mediante la búsqueda por palabras claves y rango de búsqueda en las bases de datos seleccionadas, (2) estudios irrelevantes fueron excluidos ya sea por duplicados o retiros en base al análisis de títulos y resúmenes de los estudios, (3) por consiguiente se obtuvieron estudios candidatos por cada base de datos seleccionada. (4) Se evaluó los estudios candidatos en base a la lectura de texto completa y (5) por último se obtuvieron los estudios primarios (seleccionados).

\section{Informe de la Revisión}

Esta sección representa los resultados y la discusión de esta revisión con el fin de responder a las preguntas de investigación. 


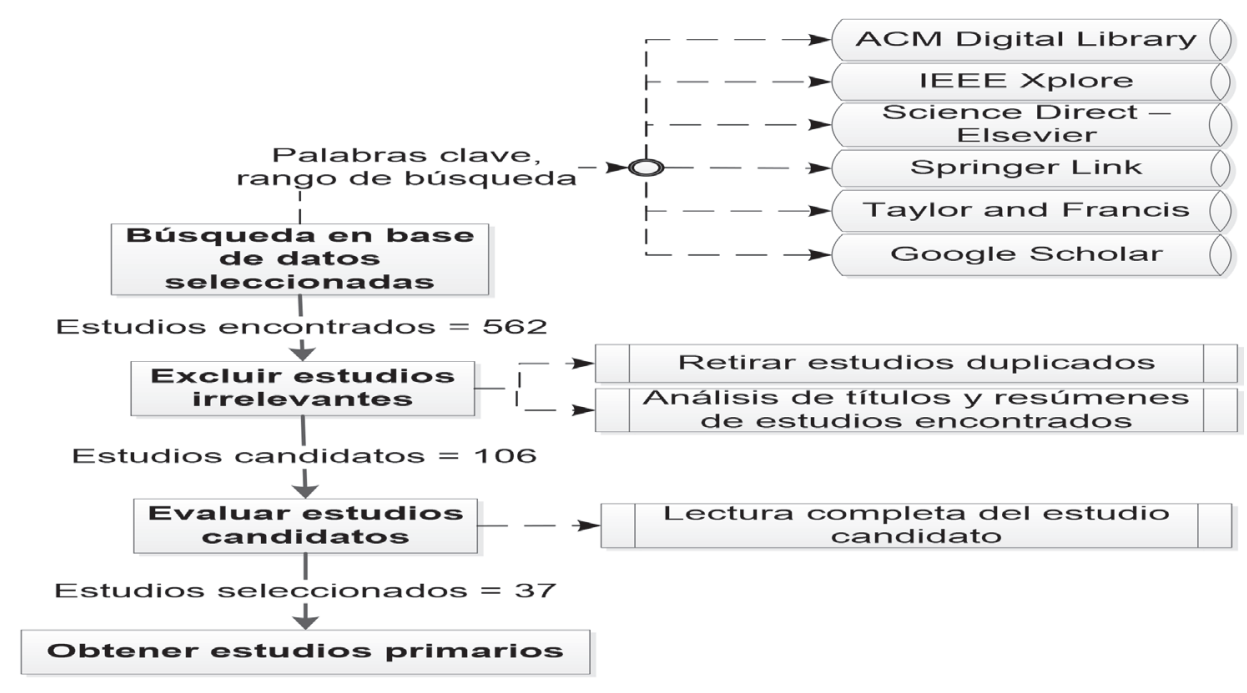

Figura 2 - Conducción de la Revisión.

\subsection{Síntesis de Datos}

En la Tabla 1, se muestran la cantidad de estudios recuperados a través de las palabras claves de búsqueda de las diferentes bases de datos seleccionadas. La primera columna muestra las fuentes de búsquedas utilizadas (base de datos seleccionadas). La segunda columna muestra el resultado de la selección inicial de artículos obtenidos en base a la búsqueda de palabras claves realizada en cada una de las bases de datos seleccionadas. La tercera columna muestra los artículos candidatos que son los números de artículos que fueron seleccionados después de la exclusión de estudios irrelevantes. La cuarta columna muestra los artículos seleccionados que representa el número de artículos seleccionados después evaluarlos en base a la lectura de texto completa. Para identificar los artículos primarios seleccionados, revisar "Anexo: Relación de Artículos Primarios”.

\begin{tabular}{llll}
\hline Fuente & Artículos encontrados & Candidatos & Seleccionados \\
\hline ACM Digital Library & 41 & 10 & 2 \\
\hline IEEE Xplore & 21 & 21 & 5 \\
\hline ScienceDirect-Elsevier & 6 & 4 & 4 \\
\hline Springer Link & 13 & 6 & 1 \\
\hline Taylor and Francis & 5 & 0 & 0 \\
\hline Google Scholar & 476 & 65 & 25 \\
\hline Total & 562 & 106 & 37 \\
\hline
\end{tabular}

Tabla 1 - Estudios seleccionados 


\subsection{Resultados}

Se busca dar respuesta a las siguientes preguntas:

\section{¿Qué factores afectan la precisión de la estimación del método de Puntos de Caso de Uso?}

Para responder a esta pregunta se ha realizado un análisis de todos los artículos seleccionados y se ha identificado los factores que afectan la precisión del método de Puntos de Caso de Uso en cuanto a la estimación del esfuerzo, los cuales se muestran en la Tabla 2. Lo mostrado en la columna "Estudios Seleccionados" hace referencia al "Anexo: Relación de Artículos Primarios".

\begin{tabular}{|c|c|c|c|}
\hline Factores & $\begin{array}{l}\text { Cantidad de } \\
\text { Artículos } \\
\text { Seleccionados }\end{array}$ & Porcentaje & Estudios Seleccionados \\
\hline Factores ambientales & 11 & $13,92 \%$ & $\begin{array}{l}{[\mathrm{A} 3][\mathrm{A} 13][\mathrm{A} 18][\mathrm{A} 2 \mathrm{O}][\mathrm{A} 22][\mathrm{A} 23]} \\
{[\mathrm{A} 24][\mathrm{A} 25][\mathrm{A} 32][\mathrm{A} 36][\mathrm{A} 37]} \\
\end{array}$ \\
\hline Evaluacion de complejidad de casos de uso & 10 & $12,66 \%$ & $\begin{array}{l}{[\mathrm{A} 2][\mathrm{A} 4][\mathrm{A} 12][\mathrm{A} 19][\mathrm{A} 21][\mathrm{A} 24]} \\
{[\mathrm{A} 27][\mathrm{A} 28][\mathrm{A} 29][\mathrm{A} 33]}\end{array}$ \\
\hline Falta de estandarización de casos de uso & 8 & $10,13 \%$ & $\begin{array}{l}{[\mathrm{A} 1][\mathrm{A} 4][\mathrm{A} 6][\mathrm{A} 11][\mathrm{A} 12][\mathrm{A} 15]} \\
{[\mathrm{A} 25][\mathrm{A} 27]}\end{array}$ \\
\hline Factores técnicos & 7 & $8,86 \%$ & $\begin{array}{l}{[\mathrm{A} 18][\mathrm{A} 20][\mathrm{A} 23][\mathrm{A} 24][\mathrm{A} 25]} \\
{[\mathrm{A} 36][\mathrm{A} 37]}\end{array}$ \\
\hline Contabilizacion de transacciones & 7 & $8,86 \%$ & $\begin{array}{l}{[\mathrm{A} 11][\mathrm{A} 27][\mathrm{A} 28][\mathrm{A} 29][\mathrm{A} 31]} \\
{[\mathrm{A} 34][\mathrm{A} 37]}\end{array}$ \\
\hline Granularidad de casos de uso & 6 & $7,59 \%$ & {$[\mathrm{~A} 1][\mathrm{A} 4][\mathrm{A} 9][\mathrm{A} 15][\mathrm{A} 17][\mathrm{A} 28]$} \\
\hline $\begin{array}{l}\text { Relación lineal entre el tamaño del } \\
\text { software y el esfuerzo }\end{array}$ & 6 & $7,59 \%$ & {$[\mathrm{~A} 5][\mathrm{A} 10][\mathrm{A} 14][\mathrm{A} 30][\mathrm{A} 35][\mathrm{A} 37]$} \\
\hline Factor de productividad & 6 & $7,59 \%$ & {$[\mathrm{~A} 2][\mathrm{A} 3][\mathrm{A} 5][\mathrm{A} 7][\mathrm{A} 8][\mathrm{A} 35]$} \\
\hline Clasificacion de actores & 5 & $6,33 \%$ & {$[\mathrm{~A} 24][\mathrm{A} 27][\mathrm{A} 28][\mathrm{A} 33][\mathrm{A} 37]$} \\
\hline $\begin{array}{l}\text { Requerimientos no funcionales en casos } \\
\text { de uso }\end{array}$ & 4 & $5,06 \%$ & {$\left[\mathrm{~A}_{4}\right]\left[\mathrm{A}_{5}\right][\mathrm{A} 16]\left[\mathrm{A}_{3} \mathrm{O}\right]$} \\
\hline $\begin{array}{l}\text { Diferente definición del término } \\
\text { transaccion de caso de uso }\end{array}$ & 2 & $2,53 \%$ & {$[\mathrm{~A} 26][\mathrm{A} 29]$} \\
\hline $\begin{array}{l}\text { Falta de definición de estandarizacion de } \\
\text { parametros }\end{array}$ & 1 & $1,27 \%$ & {$[\mathrm{~A} 2]$} \\
\hline Volatibilidad de requerimientos & 1 & $1,27 \%$ & [A25] \\
\hline Inclusión data histórica & 1 & $1,27 \%$ & {$[\mathrm{~A} 7]$} \\
\hline $\begin{array}{l}\text { Modelo de proceso de desarrollo de } \\
\text { software aplicado }\end{array}$ & 1 & $1,27 \%$ & {$[\mathrm{~A} 12]$} \\
\hline Tipo de Proyecto & 1 & $1,27 \%$ & [A25] \\
\hline Framework de software seleccionado & 1 & $1,27 \%$ & [A25] \\
\hline $\begin{array}{l}\text { No aborda cambios en requerimientos o } \\
\text { casos de uso }\end{array}$ & 1 & $1,27 \%$ & [A36] \\
\hline
\end{tabular}

Tabla 2 - Factores que afectan precisión del método de Puntos de Caso de Uso 


\begin{tabular}{|c|c|c|}
\hline $\begin{array}{l}\text { Año de } \\
\text { Publicación }\end{array}$ & Trabajo futuro & $\begin{array}{l}\text { Estudios } \\
\text { Seleccionados }\end{array}$ \\
\hline \multirow{2}{*}{2010} & $\begin{array}{l}\text { Validar resultados de estudio empírico contra Puntos de } \\
\text { Caso de Uso estándar }\end{array}$ & [A28] \\
\hline & Revisión de la estandarizacin de casos de uso & [A12] \\
\hline 2011 & Testear en proyectos con más de 5000 horas & {$[\mathrm{A} 5]$} \\
\hline \multirow{6}{*}{2013} & Factores ambientales y técnicos deben ser actualizados & [A35] \\
\hline & $\begin{array}{l}\text { Puntos de Caso de Uso debe manejar casos de usos de } \\
\text { más de } 7 \text { transacciones }\end{array}$ & [A35] \\
\hline & Pesos de casos de usos deben ser calibrados & {$[\mathrm{A} 35]$} \\
\hline & Expandir experimento con mínimo 20 proyectos & [A25] \\
\hline & Expandir experimento con más de 14 proyectos & [A20] \\
\hline & Considerar efecto de diversos factores de complejidad & {$[\mathrm{A} 32]$} \\
\hline \multirow[b]{2}{*}{2014} & Trabajar con casos de uso con al menos 21 transacciones & [A29] \\
\hline & $\begin{array}{l}\text { Incluir en el proceso de estimación los casos de uso } \\
\text { include y extend }\end{array}$ & [A29] \\
\hline
\end{tabular}

Tabla 3 - Futuros Trabajos

¿Cuáles son los futuros trabajos a realizar para mejorar el método de Puntos de Caso de Uso?

En base a la revisión de todos los artículos seleccionados se han identificado los futuros trabajos relevantes que aportan en el perfeccionamiento del método de Puntos de Caso de Uso en cuanto a la precisión de la estimación del esfuerzo, los cuales se muestran en la Tabla 3 .

\subsection{Discusiones}

Esta sección provee discusiones en respuesta a los resultados obtenidos en la sección 4.2.

\section{Discusión 1: Acerca de los factores que afectan la precisión de la estimación del método de Puntos de Caso de Uso}

En referencia a los resultados obtenidos en la Tabla 2, se ha identificado 5 factores principales que afectan la precisión de la estimación del método de Puntos de Caso de Uso, los cuales son: Factores ambientales, Evaluación de complejidad de casos de uso, Falta de estandarización de casos de uso, Factores técnicos y Contabilización de transacciones.

En referencia a los factores ambientales y factores técnicos se han consolidado cuestionamientos relevantes a la presente revisión. Los factores ambientales y técnicos originales del método de Puntos de Caso de Uso afectan la estimación (Habib, Ali \& Atique, 2012) y se encuentran desfasados (Singh \& Sahoo, 2012), por lo que se requiere una reorganización de éstos (Ribeiro, Modesto \& Santos, 2012). Además, una pequeña variación en el valor del peso de los factores ambientales incrementará 
en demasía los Puntos de Caso de Uso en cuanto al tamaño y, por tanto, el esfuerzo total del proyecto. Incluso un pequeño ajuste, por medio punto, variará el resultado final en un 40\% (Anukula \& Perumal, 2012). Los factores ambientales y técnicos son propensos al sesgo influenciado por la experiencia del estimador (Alves, Valente \& Nunes, 2013) y no presentan estandarización (Ayyıldız, Koçyiğit \& Peker, 2013). Azzeh propone la inclusión de 3 nuevos factores ambientales con el fin de ayudar a obtener mayor precisión en los resultados (Azzeh, 2013). Asimismo, Jha, Jena y Malu introducen 6 nuevos factores ambientales a fin de ayudar a obtener mayor precisión en los resultados (Jha, Jena \& Malu, 2014). Por su parte, Kirmani y Wahid realizan una revisión del método de Puntos de Caso De Uso original introduciendo un nuevo factor ambiental y un nuevo factor técnico con el fin de ayudar a obtener mayor precisión en los resultados (Kirmani \& Wahid, 2015). Por consiguiente, se resalta la necesidad de refinar los parámetros usados en los factores ambientales y técnicos los cuales están directamente relacionados a las estimaciones calculadas usando el método de Puntos de Caso de Uso (Kirmani \& Wahid, 2015).

Con respecto a la evaluación de la complejidad de los casos de uso, según Nassif, Capretz y Ho necesita calibración (Nassif, Capretz \& Ho, 2010), por lo que se debe estandarizar el nivel de complejidad de cada caso de uso en función de sus escenarios principales, transacciones y cuadros de diálogo (Remón \& Thomas, 2010), tomando en cuenta que la complejidad de un caso de uso puede variar mucho, dependiendo de cuál diseño es utilizado (Felipe et al., 2014). En los estudios de Nassif, Capretz y Ho se indican que uno de los principales inconvenientes es la ausencia de calibración cuando se clasifica la complejidad de casos de uso; por ejemplo, un caso de uso de 4 transacciones tiene dos veces el peso de un caso de uso que tiene 3 transacciones (Nassif, 2010) (Nassif, Capretz \& Ho, 2014). Kamal y Ahmed indican que un problema importante con los casos de uso es la evaluación de la complejidad del caso de uso; por ejemplo en un típico CRUD (Create, Replace, Update, Delete), sería correcto considerarlo como un caso de uso con 4 escenarios así como un caso de uso con un solo escenario (Kamal \& Ahmed, 2011). Por último en los estudios de "Kirmani y Wahid” y "Park, Moon y Kim”, se propone una nueva categorización en la complejidad de casos de uso agregando una categoría más a las ya existentes (Kirmani \& Wahid, 2015) (Park, Moon \& Kim, 2016).

Con respecto a la falta de estandarización de casos de uso se ha determinado que genera desviaciones en la estimación del método de Puntos de Caso de Uso (Remón \& Thomas, 2010) y se debe contar con la narrativa de casos de uso para hacer efectiva tal estandarización (Bajaj \& Bathla, 2014). Popović y Bojić indican que el mayor problema es la ausencia de un estándar que precisamente defina que es un caso de uso debido a que difieren de proyecto a proyecto (Popović \& Bojić, 2012). Thomas y Remón indican que debido a las múltiples formas que existen para describir un caso de uso, el resultado del valor de los Puntos de Caso de Uso puede variar considerablemente aplicando contabilización por transacciones u escenarios (Thomas \& Remón, 2011). Además de haber múltiples formas de escribir o estructurar casos de uso, las diferentes variaciones del estilo de casos de uso pueden dificultar la medida de la complejidad de un caso de uso (Chaudhary \& Khatoon, 2015). 
Con respecto a la contabilización de transacciones se ha determinado que la efectividad del método de Puntos de Caso de Uso depende principalmente de la manera en que las transacciones de casos de uso son contabilizadas (Jena \& Mishra, 2014). Asimismo en los estudios "Kirmani y Wahid” y "Park, Moon y Kim" se mencionan que el criterio para contabilizar el número de transacciones aún no está claro (Kirmani \& Wahid, 2015) (Park, Moon \& Kim, 2016). Nunes indica que se requiere una diferenciación entre una transacción de caso de uso y un escenario de caso de uso, ya que una transacción puede contener más de un escenario y viceversa (Nunes, 2010). Thomas y Remón indican que existe ausencia de una norma que permita realizar el conteo de transacciones u escenarios con lo cual se tiene una influencia directa en la estimación (Thomas \& Remón, 2011). Ochodek, Nawrocki y Kwarciak indican que algunos autores sugieren que contar transacciones es equivalente a contar pasos, lo cual en la práctica no siempre es verdad. La diferencia entre el número de pasos y el número de transacciones impacta el valor de los puntos de casos de uso (Ochodek, Nawrocki \& Kwarciak, 2011).

En base a los aportes ya descritos se hace necesario reorganizar los factores ambientales y técnicos. Sin embargo hace también falta conseguir una estandarización en cuanto a la descripción de los casos de uso, siendo esto muy importante ya que al estandarizar correctamente un caso de uso se evita más imprecisiones al momento de contabilizar transacciones. Por último en cuanto a la evaluación de la complejidad de casos de uso se ha podido determinar que tiene una gran dependencia de la contabilización de transacciones. Analizando los diferentes estudios, se ha podido inferir que los factores que sirven como entrada para la aplicación del método de Puntos de Caso de Uso son: (1) una buena aplicación de los casos de usos que está relacionada con la estandarización y granularidad de casos de uso, y (2) una correcta contabilización de transacciones de casos de uso. Lo expuesto, es punto de partida para luego calcular los siguientes pasos del método de Puntos de Caso de Uso: clasificación de los actores, complejidad de casos de uso, evaluación de factores ambientales y técnicos, obtención de los Puntos de Caso de Uso y por último la obtención del esfuerzo en horas.

\section{Discusión 2: Acerca de los futuros trabajos a realizar para mejorar el método de Puntos de Caso de Uso}

En base a los resultados obtenidos en la Tabla 3, se ha podido identificar que la mayoría de los autores hacen referencia a continuar con la afinación del método de Puntos de Casos de Uso haciendo uso de más proyectos y utilizando casos de uso con mayor cantidad de transacciones dentro de sus investigaciones (Kashyap, Shukla \& Misra, 2014) (Nunes, 2010) (Alves, Valente \& Nunes, 2013) (Ayyldı, Koçyiğit \& Peker, 2013) (Nassif, Capretz \& Ho, 2011) (Nassif, Ho \& Capretz, 2013). Otros trabajos futuros restantes como una estandarización de casos de uso (Remón \& Thomas, 2010), calibración de los pesos de casos de uso (Nassif, Ho \& Capretz, 2013), consideración de diversos factores de complejidad (Azzeh, 2013) y actualización de los factores ambientales y técnicos (Nassif, Ho \& Capretz, 2013); ya han sido evidenciados como materia de estudio por diversos autores en la presente investigación. 


\section{Anexo: Relación de Artículos Primarios}

\begin{tabular}{|c|c|c|}
\hline Núm. & Título & Autor \\
\hline$A 1$ & $\begin{array}{l}\text { A comparative evaluation of effort estimation methods in the } \\
\text { software life cycle }\end{array}$ & (Popović \& Bojić, 2012) \\
\hline A2 & A Comparative Study of Three Test Effort Estimation Methods & (Felipe, et al., 2014) \\
\hline A3 & $\begin{array}{l}\text { A hybrid model for estimating software project effort from Use } \\
\text { Case Points }\end{array}$ & (Azzeh \& Nassif, 2016) \\
\hline A4 & $\begin{array}{l}\text { A Proposed Framework for Use Case based Effort Estimation } \\
\text { using Fuzzy Logic: Building upon the outcomes of a Systematic } \\
\text { Literature Review }\end{array}$ & (Kamal \& Ahmed, 2011) \\
\hline A5 & $\begin{array}{l}\text { A Regression Model with Mamdani Fuzzy Inference System for } \\
\text { Early Software Effort Estimation Based on Use Case Diagrams }\end{array}$ & (Nassif, Capretz \& Ho, 2011) \\
\hline A6 & A Tool to Evaluate the Performance of UCP & (Bajaj \& Bathla, 2014) \\
\hline A7 & $\begin{array}{l}\text { Algorithmic Optimisation Method for Improving Use Case Points } \\
\text { Estimation }\end{array}$ & (Silhavy \& Prokopova, 2015) \\
\hline$A 8$ & $\begin{array}{l}\text { An Application of Classification and Class Decomposition to Use } \\
\text { Case Point Estimation Method }\end{array}$ & (Azzeh \& Banitaan, 2015) \\
\hline A9 & $\begin{array}{l}\text { An Elementary-Function-Based Refinement Method for Use } \\
\text { Cases to Improve Reliability of Use Case Points }\end{array}$ & (Heo, Seo \& Baik, 2015) \\
\hline A10 & $\begin{array}{l}\text { An Empirical Study on the Estimation of Software Development } \\
\text { Effort with Use Case Points }\end{array}$ & $\begin{array}{l}\text { (Alves, Sousa, Ribeiro \& } \\
\text { Machado, 2013) }\end{array}$ \\
\hline A11 & $\begin{array}{l}\text { Análisis Comparativo de Estimación de Esfuerzo en el Desarrollo } \\
\text { de Software }\end{array}$ & (Thomas \& Remón, 2011) \\
\hline A12 & $\begin{array}{l}\text { Análisis de Estimación de Esfuerzo aplicando Puntos de Caso de } \\
\text { Uso }\end{array}$ & (Remón \& Thomas, 2010) \\
\hline$A 13$ & $\begin{array}{l}\text { Analog-Based Cost Estimation For Managing Inconsistency In } \\
\text { Software Development }\end{array}$ & (Anukula \& Perumal, 2012) \\
\hline A14 & $\begin{array}{l}\text { Analysis of task effort estimation accuracy based on use case } \\
\text { point size }\end{array}$ & $\begin{array}{l}\text { (Popovic, Bojic \& Korolija, } \\
\text { 2015) }\end{array}$ \\
\hline A15 & Analysis of Use Cases and Use Case Estimation & (Chaudhary \& Khatoon, 2015) \\
\hline$A 16$ & $\begin{array}{l}\text { Applying Requirement Based Complexity For The Estimation Of } \\
\text { Software Development And Testing Effort }\end{array}$ & (Sharma \& Kushwaha, 2012) \\
\hline$A 17$ & $\begin{array}{l}\text { Applying Systems Engineering Modeling Language (SysML) to } \\
\text { System Effort Estimation Utilizing Use Case Points }\end{array}$ & (Bone \& Cloutier, 2011) \\
\hline$A 18$ & $\begin{array}{l}\text { Avaliação da Importância dos Fatores Técnicos e Ambientais do } \\
\text { Método Pontos por Caso de Uso com Base no Método AHP }\end{array}$ & $\begin{array}{l}\text { (Ribeiro, Modesto \& Santos, } \\
\text { 2012) }\end{array}$ \\
\hline A19 & Calibrating use case points & (Nassif, Capretz \& Ho, 2014) \\
\hline A2O & $\begin{array}{l}\text { Comparison of Three Software Effort Estimation Methodologies } \\
\text { with Case Study }\end{array}$ & $\begin{array}{l}\text { (Ayyıldız, Koçyiğit \& Peker, } \\
\text { 2013) }\end{array}$ \\
\hline$A 21$ & $\begin{array}{l}\text { Enhancing Use Case Points Estimation Method Using Soft } \\
\text { Computing Techniques }\end{array}$ & (Nassif, 2010) \\
\hline
\end{tabular}




\begin{tabular}{|c|c|c|}
\hline Núm. & Título & Autor \\
\hline A22 & $\begin{array}{l}\text { Estimating Software Development Effort using UML Use Case } \\
\text { Point (UCP) Method with a Modified set of Environmental } \\
\text { Factors }\end{array}$ & (Jha, Jena \& Malu, 2014) \\
\hline A23 & $\begin{array}{l}\text { Extending the UCP Model by Incorporating the Prevailing Trends } \\
\text { in Software Effort Estimation }\end{array}$ & (Habib, Ali \& Atique, 2012) \\
\hline A24 & $\begin{array}{l}\text { Impact of Modification Made in Re-UCP on Software Effort } \\
\text { Estimation }\end{array}$ & (Kirmani \& Wahid, 2015) \\
\hline A25 & $\begin{array}{l}\text { Improving software effort estimation with human-centric } \\
\text { models: a comparison of UCP and iUCP accuracy }\end{array}$ & $\begin{array}{l}\text { (Alves, Valente \& Nunes, } \\
\text { 2013) }\end{array}$ \\
\hline A26 & Improving the reliability of transaction identification in use cases & $\begin{array}{l}\text { (Ochodek, Alchimowicz, } \\
\text { Jurkiewicz \& Nawrocki, 2011) }\end{array}$ \\
\hline A27 & $\begin{array}{l}\text { Improving Use Case Point (UCP) Based on Function Point (FP) } \\
\text { Mechanism }\end{array}$ & (Park, Moon \& Kim, 2016) \\
\hline A28 & $\begin{array}{l}\text { iUCP-estimating interaction design projects with enhanced use } \\
\text { case points }\end{array}$ & (Nunes, 2010) \\
\hline A29 & $\begin{array}{l}\text { Refining the Use Case Classification for Use Case Point Method } \\
\text { for Software Effort Estimation }\end{array}$ & $\begin{array}{l}\text { (Kashyap, Shukla \& Misra, } \\
\text { 2014) }\end{array}$ \\
\hline A3O & $\begin{array}{l}\text { Regression Model for Software Effort Estimation Based on the } \\
\text { Use Case Point Method }\end{array}$ & (Nassif, Ho \& Capretz, 2011) \\
\hline A31 & Simplifying effort estimation based on Use Case Points & $\begin{array}{l}\text { (Ochodek, Nawrocki \& } \\
\text { Kwarciak, 2011) }\end{array}$ \\
\hline$A 32$ & $\begin{array}{l}\text { Software cost estimation based on use case points for global } \\
\text { software development }\end{array}$ & (Azzeh, 2013) \\
\hline A33 & Software Estimation in the Early Stages of the Software Life Cycle & (Nassif, Capretz \& Ho, 2010) \\
\hline A34 & $\begin{array}{l}\text { Survey Report on Software Cost Estimation using Use Case Point } \\
\text { Method }\end{array}$ & (Jena \& Mishra, 2014) \\
\hline A35 & $\begin{array}{l}\text { Towards an early software estimation using log-linear regression } \\
\text { and a multilayer perceptron model }\end{array}$ & (Nassif, Ho \& Capretz, 2013) \\
\hline A36 & $\begin{array}{l}\text { UML Based Object Oriented Software Development Effort } \\
\text { Estimation Using ANN }\end{array}$ & (Singh \& Sahoo, 2012) \\
\hline A37 & Use Case Point Method of Software Effort Estimation: A Review & (Kirmani \& Wahid, 2015) \\
\hline
\end{tabular}

Tabla 4 - Listado de Artículos Primarios

\section{Conclusiones}

Los trabajos de investigación de diversos autores revisados destacaron que se necesita refinación en cuanto a la estimación que provee el método de Puntos de Caso de Uso para proyectos de desarrollo de software. En base a los factores analizados que influyen en el método de Puntos de Caso de Uso se ha determinado que los punto claves en la aplicación del método de Puntos de Caso de Uso son una estandarización de casos de uso y luego una correcta contabilización de transacciones de casos de uso. Una vez realizado ello la imprecisión debería ser menor para los cálculos posteriores que conlleva el método de 
Puntos de Caso de Uso. Los estudios acerca de la afinación de las estimaciones basadas en la mejora de la clasificación de actores, evaluación de complejidad de casos de uso, cálculo de factores ambientales y técnicos o cálculo del factor de productividad son de mucha utilidad, alcanzando su mayor provecho al dar importancia tempranamente a la estandarización de casos de uso y contabilización de transacciones de casos de uso. Respecto a los trabajos futuros, con la finalidad de seguir aportando a la afinación del método de Puntos de Caso de Uso debe revisarse otros factores que afectan la estimación y trabajar en las investigaciones con una mayor cantidad de proyectos de software y también contar con proyectos de software de diferente tamaño y esfuerzo.

\section{Referencias}

Alves, R., Valente, P., \& Nunes, N. J. (2013, Junio). Improving software effort estimation with human-centric models: a comparison of UCP and iUCP accuracy. In Proceedings of the $5^{\text {th }}$ ACM SIGCHI symposium on Engineering interactive computing systems (pp. 287-296). ACM.

Alves, L. M., Sousa, A., Ribeiro, P., \& Machado, R. J. (2013, Octubre). An empirical study on the estimation of software development effort with use case points. In Frontiers in Education Conference, 2013 IEEE (pp. 101-107). IEEE.

Anukula, J. M., \& Perumal, S. M. (2012). Analog-Based Cost Estimation For Managing Inconsistency In Software Development. International Journal of Research Sciences and Advanced Engineering, 3(2), 50-54.

Ayyıldız, T. E., Koçyiğit, A., \& Peker, D. (2013). Comparison of Three Software Effort Estimation Methodologies with Case Study. In 3rd World Conference on Innovation and Computer Science (Vol. 4, pp. 257-262).

Ayyıldız, T. E., \& Koçyiğit, A. (2014). An early software effort estimation method based on use cases and conceptual classes. Journal of Software, 9(8), 2169-2173.

Azzeh, M. (2013, Marzo). Software cost estimation based on use case points for global software development. In Computer Science and Information Technology (CSIT), 2013 5th International Conference on (pp. 214-218). IEEE.

Azzeh, M., \& Banitaan, S. (2015, Diciembre). An Application of Classification and Class Decomposition to Use Case Point Estimation Method. In Machine Learning and Applications (ICMLA), 2015 IEEE 14th International Conference on (pp. 1268-1271). IEEE.

Azzeh, M., \& Nassif, A. B. (2016). A hybrid model for estimating software project effort from Use Case Points. Applied Soft Computing, 49, 981-989.

Bajaj, P., \& Bathla, D. R. (2014). A Tool to Evaluate the Performance of UCP. International Journal, 2(7).

Bone, M. A., \& Cloutier, R. (2011, Junio). 1.4. 3 Applying Systems Engineering Modeling Language (SysML) to System Effort Estimation Utilizing Use Case Points. In INCOSE International Symposium (Vol. 21, No. 1, pp. 114-127). 
Chaudhary, A., Chaudhary, N., \& Khatoon, A. (2015). Analysis of Use Cases and Use Case Estimation. International Journal Of Engineering And Computer Science, 4(3), 10791-10798.

Chavarría, A. , Bayona, S., \& Pastor, C. (2016). Aseguramiento de la Calidad en el Proceso de Desarrollo de Software utilizando CMMI, TSP y PSP. RISTI-Revista Ibérica de Sistemas e Tecnologias de Informação, (20), 62-77.

Clemmons, R. K. (2006). Project estimation with use case points. The Journal of Defense Software Engineering, 18-22.

Felipe, N. F., Cavalcanti, R. P., Maia, E. H. B., Amaral, W. P., Farnese, A. C., Tavares, L. D., ... \& de Pádua Paula Filho, W. (2014). A Comparative Study of Three Test Effort Estimation Methods. Revista Cubana de Ciencias Informáticas, 8, 1-13.

Flores-Rios, B. L., Pino, F. J., Ibarra-Esquer, J. E., González-Navarro, F. F., \& RodríguezElías, O. M. (2014). Análisis de Flujos de Conocimiento en Proyectos de Mejora de Procesos Software bajo una perspectiva multi-enfoque. RISTI-Revista Ibérica de Sistemas e Tecnologias de Informação, (14), 51-66.

Habib, M. U., Ali, M. A., \& Atique, N. (2012). Extending the UCP Model by Incorporating the Prevailing Trends in Software Effort Estimation. International Journal of Computer Applications, 59(5).

Heo, R., Seo, Y. D., \& Baik, D. K. (2015). An Elementary-Function-Based Refinement Method for Use Cases to Improve Reliability of Use Case Points. Journal of KIISE, 42(9), 1117-1123.

Jena, P. P., \& Mishra, S. (2014). Survey Report on Software Cost Estimation Using Use Case Point Method. International Journal of Computer Science \& Engineering Technology, 5(4), 280-287.

Jha, P., Jena, P. P., \& Malu, R. K. (2014). Estimating software development effort using UML use case point (UCP) method with a modified set of environmental factors. International Journal of Computer Science and Information Technologies, 5(3), 2742-2744.

Kamal, M. W., \& Ahmed, M. A. (2011). A proposed framework for use case based effort estimation using fuzzy logic: building upon the outcomes of a systematic literature review. International Journal of New Computer Architectures and their Applications (IJNCAA), 1(4), 953-976.

Karner, G. (1993). Resource estimation for objectory projects. Objective Systems SF AB, 17.

Kashyap, D., Shukla, D., \& Misra, A. K. (2014). Refining the Use Case Classification for Use Case Point Method for Software Effort Estimation. In Proceedings of International Conference on Recent Trends in Information, Telecommunication and Computing, ACEEE Association of Computer Electronics and Electrical Engineers.

Kirmani, M. M., \& Wahid, A. (2015). Impact of modification made in Re-UCP on software effort estimation. Journal of software engineering and applications, 8(06), 276. 
Kirmani, M. M., \& Wahid, A. (2015). Use case point method of software effort estimation: a review. International Journal of Computer Applications, 116(15).

Kusumoto, S., Matukawa, F., Inoue, K., Hanabusa, S., \& Maegawa, Y. (2005). Effort estimation tool based on use case points method. Osaka University.

Morgenshtern, O., Raz, T., \& Dvir, D. (2007). Factors affecting duration and effort estimation errors in software development projects. Information and Software Technology, 49(8), 827-837.

Nassif, A. B. (2010). Enhancing use case points estimation method using soft computing techniques. Journal of Global Research in Computer Science, 1(4).

Nassif, A. B., Capretz, L. F., \& Ho, D. (2010, Enero). Software estimation in the early stages of the software life cycle. In International conference on emerging trends in computer science, communication and information technology (pp. 5-13).

Nassif, A. B., Capretz, L. F., \& Ho, D. (2011). A regression model with mamdani fuzzy inference system for early software effort estimation based on use case diagrams. In Third International Conference on Intelligent Computing and Intelligent Systems (pp. 615-620).

Nassif, A. B., Ho, D., \& Capretz, L. F. (2011). Regression model for software effort estimation based on the use case point method. In 2011 International Conference on Computer and Software Modeling (Vol. 14, pp. 106-110).

Nassif, A. B., Ho, D., \& Capretz, L. F. (2013). Towards an early software estimation using log-linear regression and a multilayer perceptron model. Journal of Systems and Software, 86(1), 144-160.

Nassif, A. B., Capretz, L. F., \& Ho, D. (2014, Mayo). Calibrating use case points. In Companion Proceedings of the 36th International Conference on Software Engineering (pp. 612-613). ACM.

Nunes, N. J. (2010). iUCP-Estimating Interaction Design Projects with Enhanced Use Case Points. Task Models and Diagrams for User Interface Design, 131.

Ochodek, M., Alchimowicz, B., Jurkiewicz, J., \& Nawrocki, J. (2011). Improving the reliability of transaction identification in use cases. Information and Software Technology, 53(8), 885-897.

Ochodek, M., Nawrocki, J., \& Kwarciak, K. (2011). Simplifying effort estimation based on Use Case Points. Information and Software Technology, 53(3), 200-213.

Park, B. K., Moon, S. Y., \& Kim, R. Y. C. (2016, Febrero). Improving Use Case Point (UCP) Based on Function Point (FP) Mechanism. In Platform Technology and Service (PlatCon), 2016 International Conference on (pp. 1-5). IEEE.

Popović, J., \& Bojić, D. (2012). A comparative evaluation of effort estimation methods in the software life cycle. Computer Science and Information Systems, 9(1), 455-484.

Popovic, J., Bojic, D., \& Korolija, N. (2015). Analysis of task effort estimation accuracy based on use case point size. IET Software, 9(6), 166-173. 
Pow-Sang, J. (2004). Estudio de Técnicas basadas en Puntos de Función para la Estimación del Esfuerzo en Proyectos Software. Revista de investigación de Sistemas e Informática, 1(1), 73-82.

Recalde, O. M., \& Lopez, A. M. (2010). Estimación Basada en Casos de Uso UCP-Use Case Points (Doctoral dissertation, Tesis de Maestría. Universidad Politécnica de Madrid, Madrid).

Remón, C. A., \& Thomas, P. J. (2010). Análisis de Estimación de Esfuerzo aplicando Puntos de Caso de Uso. In XVI Congreso Argentino de Ciencias de la Computación.

Ribeiro, B. B., Modesto, D. M., \& Santos, G. (2012). Avaliação da Importância dos Fatores Técnicos e Ambientais do Método Pontos por Caso de Uso com Base no Método AHP. In CIbSE (pp. 210-223).

Saroha \& Sahu (2015). Analysis of various Software Effort Estimation Techniques. International Research Journal of Computers and Electronics Engineering (IRJCEE), 3(2), 1-7.

Satapathy, S. M., \& Rath, S. K. (2014). Use case point approach based software effort estimation using various support vector regression kernel methods. arXiv preprint arXiv:1401.3069.

Sharma, A., \& Kushwaha, D. S. (2012). Applying requirement based complexity for the estimation of software development and testing effort. ACM SIGSOFT Software Engineering Notes, 37(1), 1-11.

Silhavy, R., Silhavy, P., \& Prokopova, Z. (2015). Algorithmic optimisation method for improving use case points estimation. PloS one, $10(11)$, e0141887.

Singh, J., \& Sahoo, B. (2012). UML Based Object Oriented Software Development Effort Estimation Using ANN.

Thomas, P. J., \& Remón, C. A. (2011). Análisis comparativo de estimación de esfuerzo en el desarrollo de software. In XVII Congreso Argentino de Ciencias de la Computación.

Trendowicz, A., \& Jeffery, R. (2014). Software Project Effort Estimation. Foundations and Best Practice Guidelines for Success, Constructive Cost Model-COCOMO pags, 277-293.

Vasconcelos, J. B., Kimble, C., Carreteiro, P., \& Rocha, Á. (2017). The application of knowledge management to software evolution. International Journal of Information Management, 37(1), 1499-1506.

Wang, F., Yang, X., Zhu, X., \& Chen, L. (2009, Diciembre). Extended use case points method for software cost estimation. In Computational Intelligence and Software Engineering, 2009. CiSE 2009. International Conference on (pp. 1-5). IEEE. 